\title{
Análise da Comparativa da Utilização da Tecnologia BIM em Projeto de Residências Unifamiliares
}

\author{
Comparative analysis of the use of BIM technology in the design of single-family buildings
}

Daniel de Andrade Martins ${ }^{\dagger *}$, Adauri Silveira Rodrigues Júnior

Como citar esse artigo. Martins, DA;

Rodrigues Junior, AS. Análise . Revista

Teccen. 2019 Jul/Dez; 12 (2): 02-10.

\begin{abstract}
Resumo
O setor da construção civil cada vez mais tem buscado otimizar os seus processos, de maneira a aumentar sua qualidade e diminuir os desperdícios. Esse fator favoreceu o surgimento de novas tecnologias para a área, como a tecnologia BIM (Building Information Management), que realiza o projeto das edificações de forma tridimensional e integrando todasas etapas de modelamento e as equipes envolvidas. Entretanto, a técnica possui um investimento alto (considerando custos com software e hardware) e necessita de profissionais capacitados e bem treinados para a sua implantação, uma vez que é necessário um conhecimento técnico para explorar todas as funcionalidades desta tecnologia. Tendo isso em mente, o presente estudo buscou realizar uma análise comparativa entre as ferramentas da tecnologia BIM com o software Revit ${ }^{\circledR}$ e das ferramentas convencionais em projeto de prédios unifamiliares,tais como software AutoCAD ${ }^{\circledR}$ por meio das plantas bidimensionais. Pode-se concluirque o BIM possui aplicabilidade nos projetos de residências unifamiliares visto que possibilita o conhecimento de detalhes no projeto e, consequentemente, permite um melhor gerenciamento e execução.

Palavras-Chave: Modelamento BIM, Projeto Tridimensional, Planejamento Residencial.
\end{abstract}

\begin{abstract}
The construction industry is increasingly seeking to optimize its processes in order to increase its quality and reduce waste. This factor favored the emergence of new technologies for the area, such as BIM (Building Information Management) technology, which performs the design of buildings in a three-dimensional way and integrating all the modeling stages and the teams involved. However, the technique has a high investment (considering software and hardware costs) and needs qualified and well trained professionals for its implementation, since it is necessary a technical knowledge to explore all the functionalities of this technology. Keep this in mind,this study conducted a comparative analysis between BIM technology tools with Revit ${ }^{\circledR}$ software and conventional single-family building design tools such as AutoCAD ${ }^{\circledR}$ software using two-dimensional floor plans. It can be concluded that BIM has applicability in single-family home projects since it allows the knowledge of details in the project and, consequently, allows better management and execution.

Keywords: BIM Modeling, Three Dimensional Design, Home Planning.
\end{abstract}

\section{Introdução}

A construção civil é um setor que cada vez mais procura por inovações, a fim de otimizar as suas edificações e processos construtivos, além de atender as exigências dos consumidores por maior qualidade. Dessa forma, tem ocorrido um aprimoramento tecnológico, buscando minimizar desperdícios, melhorar a qualidade e aumentar a eficiência. A fase que merece maior destaque é a de projeto e planejamento de uma edificação, pois é nela que são definidos os principais parâmetros que serão utilizados ao longo da construção e que influenciam no orçamento, cronograma e métodos de produção.

Dentro dessa temática, uma ferramenta que vem sendo utilizada com grande frequência são as baseadas na tecnologia BIM, do inglês Building Information Modeling (Modelagem da Informação da Construção), que consiste em uma metodologia para gerenciamento de projetos de forma digital, controlando dados durante todo o ciclo de vida da construção. Com esse método é possível unir as informações e detalhes de vários projetos de construção e criar um modelo 3D da obra, muito mais próximo do resultado. Assim, os softwares passaram a ser paramétricos e tridimensionais, permitindo que as equipes pudessem trabalhar de forma integrada e colaborativa, e diminuindo a chance de erros.

Apesar disso, ainda existem barreiras para a utilização do BIM, como investimentos de implantação elevados e escassez de profissionais capacitados na área. Logo, a escolha da sua aplicação deve ser cuidadosa, para que seja aplicada adequadamente.

Afiliação dos autores: †Graduando em Engenharia Civil - Curso de Engenharia Civil, Universidade de Vassouras, Vassouras, RJ, Brasil

\$Mestre em Engenharia Civil - Curso de Engenharia Civil, Universidade de Vassouras, Vassouras, RJ, Brasil

* Email para correspondência: daniel_batmartins@hotmail.com 


\section{Objetivo}

O presente trabalho buscou realiza uma análise comparativa entre a utilização da tecnologia BIM em relação aos sistemas convencionais, através de uma revisão bibliográfica dos principais estudos sobre o tema.

\section{Objetivos específicos}

Foram definidos os seguintes objetivos específicos:

a) Estudar os principais métodos de projeto da tecnologia BIM e os métodos convencionais;

b) Selecionar estudos de aplicação das ferramentas dos dois métodos em residências unifamiliares;

c) Analisar comparativamente os resultados obtidos pelos diferentes autores;

d) Sintetizar as observações encontradas no formato de um artigo.

\section{Problemática}

Diante do crescente uso da tecnologia BIM em projetos de construções, levanta-se o questionamento: quais as vantagens e desvantagens da tecnologia BIM em relação aos métodos convencionais? Seu uso tem sido aplicado adequadamente? No caso de construções unifamiliares seu uso é benéfico e indicado?

\section{Justificativa}

O desenvolvimento desse trabalho pretende contribuir para a formação de profissionais capacitados na aplicação da tecnologia BIM na elaboração de seus projetos, podendo identificar quando seu uso é indicado. Além disso, visa disseminar suas informações para favorecer o aumento da utilização dessa tecnologia de maneira correta, a fim de obter melhores resultados. Assim, irá auxiliar o mercado da construção a obter edificações mais seguras, com baixo índice de desperdícios e elevada qualidade, além de aumentar a produtividade, beneficiando o cliente e os construtores.

\section{Revisão de literatura Tecnologia CAD}

Os avanços tecnológicos levaram a inserção de tecnologias computacionais para projetar, fabricar e montar elementos construtivos, tanto dentro quanto fora do canteiro de obras. Uma das ferramentas que é amplamente utilizada nesse sentido é o CAD (Computer Aided Design ou Desenho Auxiliado por Computador), que substituiu o trabalho feito manualmente nas pranchetas (Marinho, 2017).

Conforme Campos Neto, Taveira e Moreau (2017) e Marinho (2017), comparativamente à realização manual de projetos, o CAD geométrico trouxe melhorias na produtividade, devido a benefícios como: aumento na qualidade de informações gráficas, velocidade de desenho do projeto e padronização. Dessa forma, permitiu produções mais complexas, principalmente no que diz respeito à arquitetura, automatizando o processo de projetação, reduzindo o tempo gasto em tarefas repetitivas, fazendo com que o projetista possa desempenhar outras atividades, como planejamento e controle (Marinho, 2017).

O sistema CAD geométrico é amplamente utilizado nos escritórios de arquitetura e teve sua origem na década de 60 , voltado para fins industriais. Posteriormente, a evolução ocorreu no sentido da modelagem de produto, onde a dificuldade inicial foi de hardware, dificultando sua utilização (Martinez \& Amorim, 2010).

A tecnologia CAD pode ter duas interpretações, conforme Marinho (2017):

a) Computer Aided Drafting: se baseia no auxílio do computador na representação gráfica no processo de projeto em prancheta, a qual possui uma formação mental do espaço, onde o projetista faz diversos desenhos como plantas, cortes, elevações e perspectivas no plano para entendimento do seu objeto de projeto.

b) Computer Aided Design: as representações bidimensionais e tridimensionais possuem funções automáticas, ajudando o projetista a tomar decisões, como funções de cálculo de áreas, perímetros e volumes, até funções mais avançadas de sistemas integrados de informação.

Além disso, essa segunda interpretação ainda pode se dividir em:

a) Computer Aided Engineering (CAE): representa os projetos de engenharia e seus sistemas são capazes de executar cálculos, análises, dimensionamentos e simulações de engenharia;

b) Computer Aided Architectural Design (CAAD): auxilia os projetos de arquitetura, onde os seus sistemas executam funções de desenvolvimento, avaliação e simulação da edificação em diversas etapas do projeto.

O AutoCAD (Figura 1) foi o software pioneiro em plataforma $\mathrm{CAD}$ e é o aplicativo CAD com maior uso. É comercializado pela Autodesk desde 1982, e nas versões atuais, permite ao usuário a realização de desenhos $2 \mathrm{D}$ com a utilização de gráficos vetoriais e em 3D com modelagem de superfícies sólidas. No AutoCAD também é possível realizar customizações 


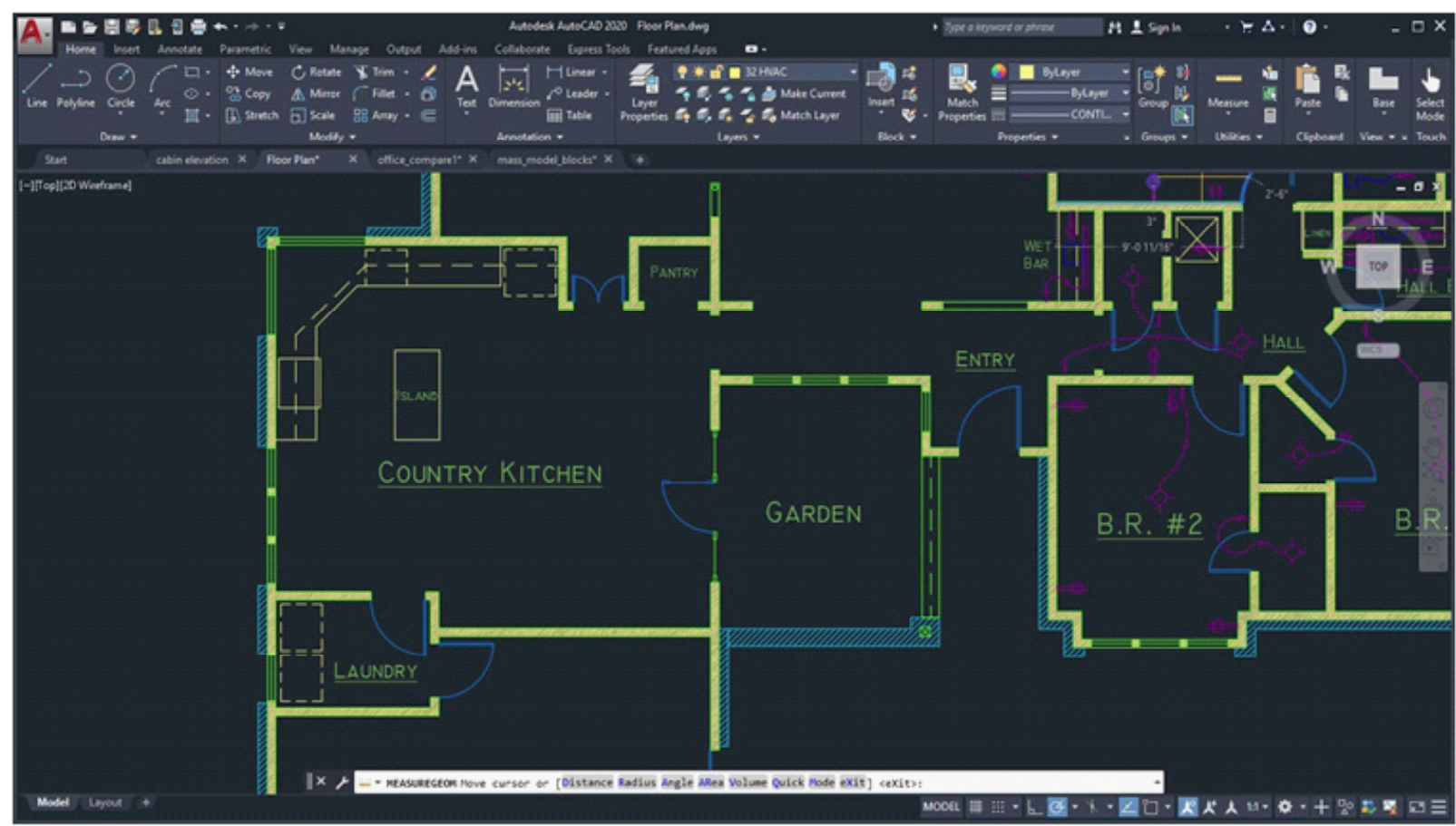

Figura 1. Interface do AutoCAD

Fonte: Teixeira (2016, p. 25)

de funções, programáveis em "AutoLISP”, que é uma variação do Visual Basic (Teixeira, 2016).

A maior parte das empresas que trabalham com compatibilização de projetos utiliza o processo tradicional, através da sobreposição de diferentes disciplinas em umúnico arquivo no AutoCAD desligando os componentes que não fazem parte dos sistemas, determinando a olho nu as possíveis interferências (Silva, 2018).

\section{Tecnologia BIM}

\section{Histórico}

De acordo com Pires (2018), a ideia do BIM foi primeiramente apresentada em 1975, por Charles M. "Chuck" Eastman no extinto AIA Journal, em um trabalho denominado "Building Description System". Segundo Pereira (2017), a tecnologia BIM começou a ser desenvolvida no final da década de 80, pelo arquiteto Jerry Laisern da Universidade de Princenton (EUA), especialista em Tecnologia da Informação (TI). Ele deu origem à IAI (Internacional Alliance for Interoperability) em razão de suas pesquisas na área de TI e interoperabilidade.

Porém, o sistema só começou a ser adotado em larga escala por volta de 2003, quando companhias como a Bentley, Autodesck, Optira e Commonpoint, durante a Conferência de Construção em Seattle (EUA), apresentaram à GSA (General Services Administration) a modelagem em 3D parametrizada, a integração com o cronograma e asanálises energéticas das edificações. Tal demonstração inspirou a implementação de um plano de adoção do BIM na construção pública americana e resultou na adoção em larga escala do BIM pelas empresas de projeto, construção e fornecimento de material na América do Norte (Pereira, 2017).

O conceito surgiu a partir da necessidade de gerar modelos representativos diferentes das técnicas tradicionais de CAD disponíveis, de modo que esse modelo fosse a interação de elementos individuais com características geométricas, físicas, entre outras. No modelo BIM todos os profissionais que trabalham concomitantemente nos projetos, compartilham de um modelo único, onde as informações são processadas no mesmo instante em que são lançadas, dessa forma, quando um projeto é modificado todos os demais recebem essa informação (Silva \& Comparim, 2016).

Apesar de ser amplamente utilizado nos EUA, Inglaterra e outros países, no Brasil mostra que a disseminação da ferramenta ainda está restrita aos escritórios de arquitetura e em fase bastante preliminar. O que dificulta a utilização do sistema são os problemas de interoperabilidade, ou seja, a correta troca das informações entre os softwares (Martinez \& Amorim, 2010).

Mais atualmente, Pereira (2017) aponta que, apesar das dificuldades de implantação, essa plataforma 
já começou a ser adotada por vários profissionais das áreas de orçamentos, arquitetura, estrutura, instalações prediais e de vedação.

Entretanto, ainda não existem em nosso país leis específicas ou decretos relativos ao uso do BIM, de âmbito federal. Existe uma comissão especial de estudo voltada ao BIM, a ABNT/CEE134 - Modelagem de Informação da Construção, definida em 2010, com um grupo de trabalho dedicado ao estudo da normalização de conteúdo para objetos virtuais e respectivas bibliotecas a serem utilizados nos sistemas BIM, todavia, nenhum documento ainda foi publicado (Brito, 2017).

\section{Definição}

Existem diversas definições para o termo BIM. De acordo com Aparecida (2018), indicam que o termo BIM - sigla americana para Building Information Modeling - foi utilizado inicialmente por Charles Eastman, um professor da Georgia Tech School of Architecture, que o definiu como um modelo virtual responsável por representar um produto, a partir do fluxo de informações do desenvolvimento do projeto deste produto.

Para Kymmell (2008) apud Brito (2017), o BIM é uma simulação de projeto, compondo-se de um modelo tridimensional e seus componentes, ligados a todas as informações necessárias e relacionadas ao planejamento, construção ou operação do projeto.

De maneira mais abrangente, Marinho (2017) propõe que o BIM pode ser entendido como um conjunto de políticas, processos e tecnologias que, combinados, são capazes de gerenciar, coordenar e planejar o processo de projeto de uma edificação ou instalação, além de ser capaz de realizar ensaios prévios de desempenho, gerenciar e armazenar informações e dados, durante todo o ciclo de vida da obra, desde a concepção e a conceituação da ideia, desenvolvimento do projeto, a construção, e também após a obra pronta, na sua fase de utilização. Portanto, trata-se de uma plataforma de trabalho, que é baseada em modelos, ou seja, uma representação digital multidimensional das características físicas e funcionais de uma edificação ou instalação.

O BIM é um sistema inteligente caracterizado pela modelagem paramétrica com orientação a objetos, onde são utilizados objetos 3D inteligentes, como paredes, portas, janelas, etc., aos quais se agregam um banco de dados com diversas informações referentes a processos, produtos, custos, documentos, etc., além do próprio sistema gerar documentos de projetos como cortes e fachadas automaticamente do modelo 3D (Martinez \& Amorim, 2010).

É importante destacar que o BIM não é um software, mas um novo estágio na elaboração de projetos de construção. Muitos ainda pensam que se trata de uma ferramenta de modelagem em 3D, porém, o BIM é um conceito e uma forma de gestão da informação que aplica diferentes sistemas. É, portanto, a união da tecnologia dos softwares com uma gestão da informação eficiente e uma cultura de comunicação atuante no dia a dia (Mobus Construção, 2018).

\section{Componentes e características}

Para Pereira (2017), a representação através do BIM deve ser:

a) Digital;

b) Espacial (3D);

c) Mensurável (quantificável, dimensionável e consultável);

d) Abrangente (incorporando e comunicando a intenção de projeto, o desempenho da construção, a construbilidade, e incluir aspectos sequenciais e financeiros de meios e métodos);

e) Acessível (a toda equipe do empreendimento e ao proprietário por meio de uma interface interoperável e intuitiva);

f) Durável (utilizável ao longo de todas as fases da vida de uma edificação).

Segundo Silva e Comparim (2016), a modelagem BIM propicia ao usuário a construção do empreendimento virtual ainda na fase de projetos, tornando possível uma efetiva comunicação, troca de dados, estabelecimento de padrões e protocolos necessário para que todos os sistemas e equipes conversem entre si.

De acordo com Eastman et al. (2008) apud Soliman Junior (2015, p. 20) é uma tecnologia de modelagem caracterizada por:

a)Componentes de construção que são representados através de representações digitais inteligentes que sabem o que eles são, e podem ser associados a atributos gráficos e de dados computáveis e regras paramétricas; b)Componentes que incluem dados que descrevem como ser comportam, conforme a necessidade de análises e processos de trabalho, por exemplo, extração de quantitativos, especificação e análise energética; c)Dados consistentes e não redundantes de forma que as modificações nos dados dos componentes sejam representadas em todas as visualizações deste componente; d)Dados coordenados de forma que todas as visualizações do modelo sejam representadas de forma coordenada (Eastman et al., 2008 apud Soliman Junior, 2015, p. 20).

Dentro dessa plataforma constituída pela tecnologia BIM, conforme Pereira (2017), pode ocorrer diversas camadas de informação, conhecidas como dimensões. Um modelo pode ser 4D, 5D, 6D, 7D, até $\mathrm{nD}$, conforme o contexto da utilização. As principais 
são:

a) estão representadas graficamente as plantas do empreendimento;

b) 3D Modelo: adiciona-se a dimensão espacial ao plano, onde é possível visualizar os objetos dinamicamente. Cada componente em 3D possuí atributos e parametrização que os caracterizam como parte de uma construção virtual de fato, não apenas visualmente representativa;

c) 4D Planejamento: há a adição da dimensão tempo ao modelo, definindo quando cada elemento será comprado, armazenado, preparado, instalado, utilizado.

Organiza também a disposição do canteiro de obras, a manutenção e movimentação das equipes, os equipamentos utilizados e outros aspectos que estão cronologicamente relacionados.

d) 5D Orçamento: representa a adição do custo ao modelo, determinando quanto cada parte da obra vai custar, a alocação de recursos a cada fase do projeto e seu impacto no orçamento, o controle de metas da obra de acordo com os custos.

e) 6D Sustentabilidade: adiciona a dimensão energia ao modelo, quantificando e qualificando a energia utilizada na construção, a energia a ser consumida no seu ciclo de vida e seu custo, em paralelo a $5^{\circ}$ dimensão. A energia, neste caso, pode estar diretamente relacionada ao impacto físico do projeto no meio em que este está inserido.

f) 7D Gestão de Instalações: a dimensão de operação ao modelo, onde o usuário final pode extrair informações de como o empreendimento como um todo funciona, suas particularidades, quais os procedimentos de manutenção em caso de falhas ou defeitos.

\section{Softwares}

Com a popularização cada vez maior da tecnologia BIM, há uma enorme variedade de softwares que abrangem essa plataforma, sendo cada um deles dedicado às fases específicas do projeto. A Building Smart, organização internacional que visa melhorar o intercâmbio de informações entre aplicativos de softwares utilizados na construção, define que para ser considerado BIM, a aplicação deve importar e/ou exportar em formato IFC (Industry Foundation Classes) (Pires, 2018).

Conforme Pires (2018), alguns dos principais softwares são:

a) Revit (Autodesk): modelagem, compatibilização e quantitativos;

b) ArchiCAD (GRAPHISOFT): modelagem, compatibilização e quantitativos;

c) AECOSim Building Designer (Bentley Systems): modelagem e quantitativos;

d) Vectorworks (NEMETSCHEK): modelagem e quantitativos;

e) TeklaStructure (Trimble): modelagem; detalhamento; estrutural e quantitativos;

f) SolibriModel Checker (NEMETSCHEK): compatibilização e quantitativos;

g) Tekla BIM Sight (Trimble): compatibilização;

h) Navisworks (Autodesk): planejamento, compatibilização e quantitativos;

i) Synchro pro (Bentley Systems): planejamento;

j) Vico Office (Trimble): planejamento e orçamento;

k) ArchiBUS (ArchiBUS): operação e manutenção.

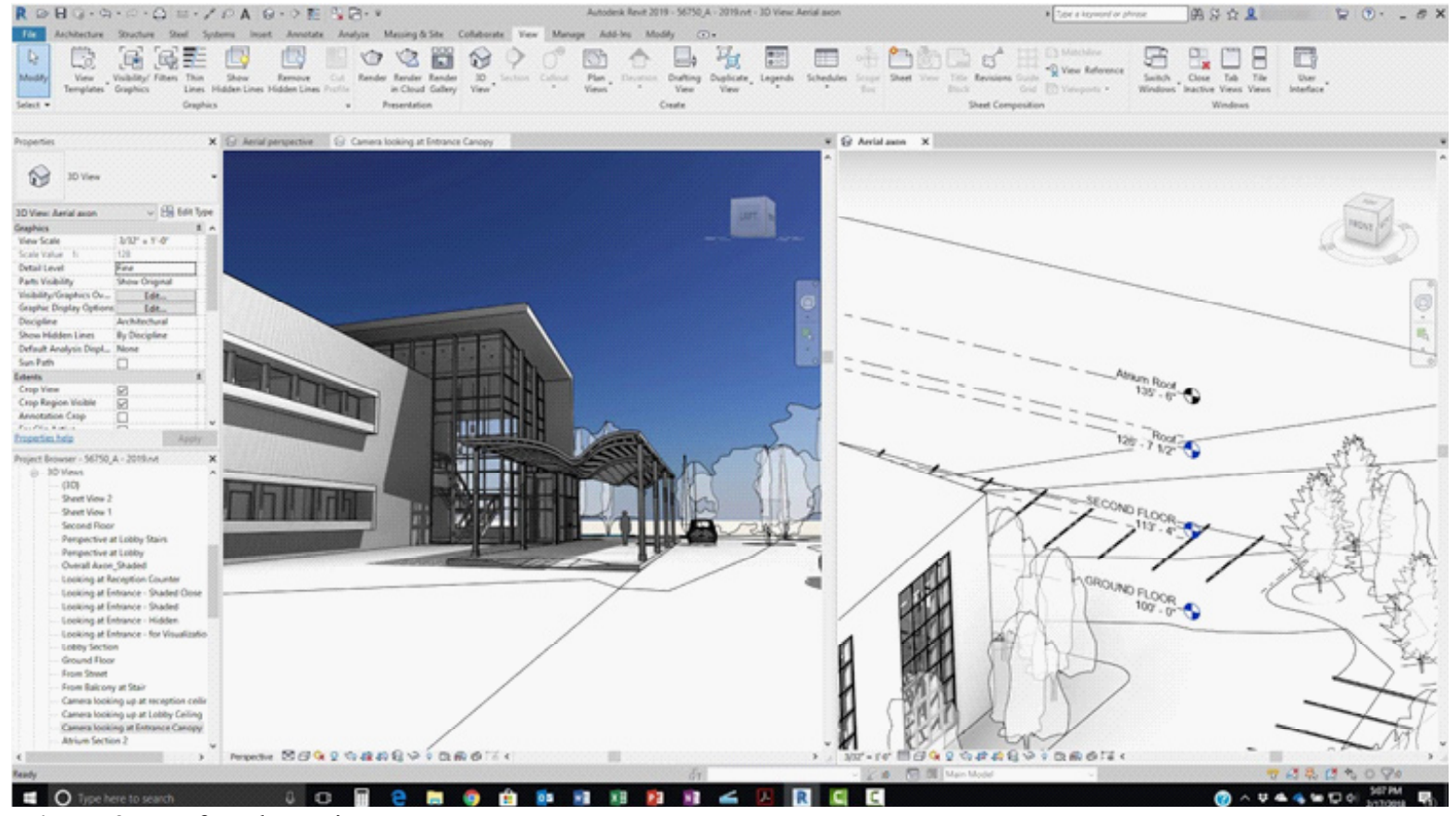

Figura 2. Interface do Revit 
O Revit(Figura 2) é considerado o programa mais conhecido do mercado, introduzido pela Autodesk em 2002. Ele é uma família de produtos integrados que inclui o Revit Architecture, Revit Structure e Revit MEP (Perini, 2017).

\section{Vantagens e Desvantagens}

As vantagens da plataforma BIM podem englobar todo o ciclo de vida da edificação, desde os estudos e análises de viabilidade do empreendimento até o a demolição do edifício. A plataforma permite a simulação $3 \mathrm{D}$ da edificação pronta, bem como a construção virtual do projeto, apresentando informações mais confiáveis e, por esses motivos, consegue viabilizar maiores condições de economia de tempo e de dinheiro (Aparecida, 2018).

As principais vantagens, conforme Pereira (2017), são:

a) Diminuição de erros de desenho;

b) Facilidade nas modificações de projeto as quais são realizadas automaticamente em todo o modelo;

c) Construção mais económica e consistente;

d) Mais ajustes na execução;

e) Quantitativos de materiais mais precisos;

f) Visualização 3D da estrutura;

g) Melhor compreensão visual do projeto;

h) Melhor preparação do projeto;

i) Modelação de objetos com definição das suas propriedades físicas;

j) Facilidade na obtenção de documentos de construção (plantas, cortes, detalhes, alçados, entre outros);

k) A estrutura é modelada uma única vez, podendo ser usada nas várias especialidades e fases do projeto;

1) Consolidação da informação do projeto apenas num único ficheiro informático;

m) Elevado nível da produtividade;

n) Facilidade de concepção e percepção das várias

fases de construção;

o) Simplifica intervenções futuras no projeto.

Outro benefício desse sistema é referente ao levantamento de quantitativos automáticos que a ferramenta pode gerar, tendo um ganho significativo de tempo no processo de planejamento e orçamentação de um empreendimento. Sendo assim, caso haja uma modelagem bem-feita, pode-se eliminar as possíveis incoerências entre projetos, proporcionando a um levantamento e quantitativos mais preciso e eficiente, fazendo com que as informações que chegam ao canteiro de obras sejam mais confiáveis para o projeto como um todo (Marinho, 2017).

Algumas desvantagens apresentadas por Pereira (2017) para a aplicação desse sistema são: a) Necessidade de aquisição de software;

b) Mudança de mentalidades;

c) Necessidade de formação dos futuros utilizadores;

d) Necessidade de computadores mais potentes e com mais memória.

Semelhantemente, Silva e Comparim (2016) apontam como desvantagens:

a) Custo inicial elevado para aquisição dos programas;

b) Ainda há poucos clientes que pagam pelo serviço;

c) Falta de comunicação com os softwares de projetos complementares;

d) Poucos profissionais habilitados para a elaboração de projetos em 3D.

\section{Comparativo entre a tecnologia CAD e a tecnologia BIM}

Por seus diferentes métodos de aplicação, podemos claramente diferenciar as tecnologias CAD e BIM. A plataforma CAD pode gerar desenhos de construções abstratas e são representadas apenas por elementos geométricos, como linhas, arcos e polígonos, sem informações úteis que ajudem na distinção desses elementos, dificultando o processo de quantificação e classificação desses componentes como elementos construtivos. Além disso, os desenhos feitos em CAD se baseiam em coordenadas geométricas, que unidos, podem representar elementos distintos, como paredes, lajes, vigas, etc., entretanto, a modificação de algum elemento implica em diversas alterações manuais, que podem acabar requerendo uma grande quantidade de tempo (Marinho, 2017).

Enquanto isso, ainda segundo Marinho (2017), o BIM consiste em elementos de geometrias construtivas em três dimensões, contendo informações e atributos que possam the conferir identidade, como uma parede, por exemplo. Essa diferença faz com que a plataforma BIM possua informações em maiores quantidades e mais precisas do que os desenhos feitos em CAD. Além disso, devido a sua modelagem paramétrica, quando se realiza uma alteração em algum elemento de um projeto no BIM, todo o modelo é atualizado, dispensando alterações manuais. desenvolvimento de projetos baseado apenas em documentos e desenhos (CAD), e a plataforma BIM, considerando a variação da capacidade de influenciar os custos e a qualidade de um empreendimento no decorrer das diversas fases do projeto. A ferramenta BIM demanda um maior esforço nas fases iniciais do desenvolvimento de um projeto do que o CAD, entretanto, nas demais fases, pode-se perceber menos esforço e menores impactos.

O conceito BIM prevê a construção em ambiente 3D virtual de objetos característicos e não da sua 

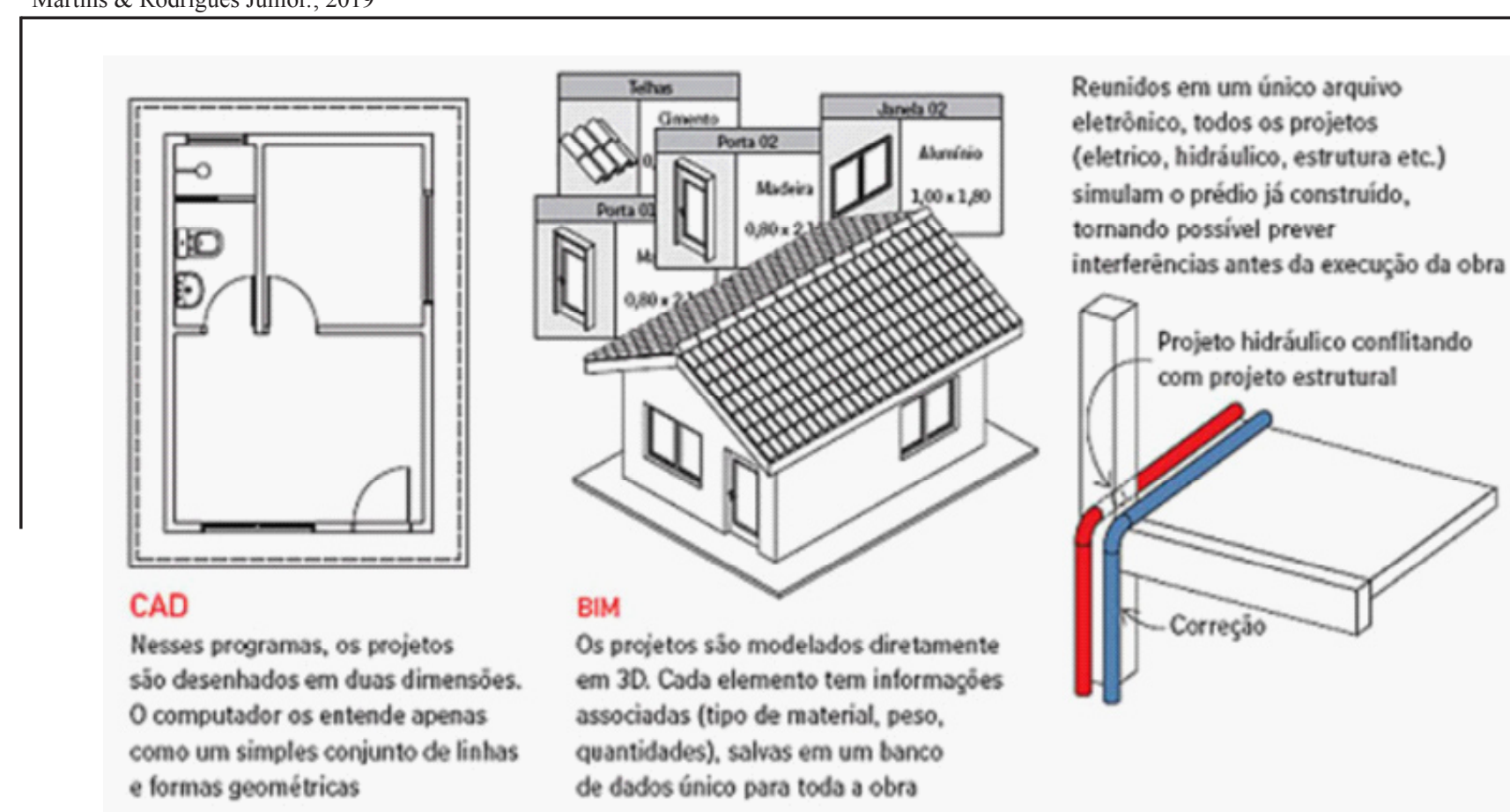

Figura 3. Diferenças entre as tecnologias CAD e BIM

representação. Tais objetos chamados de objetos inteligentes (objetos paramétricos de construção), apresentam, além das propriedades espaciais associadas à sua representação, propriedades intrínsecas aos mesmos. Se utilizarmos o objeto "porta" a título de exemplo, teremos nos softwares CAD a representação geométrica do objeto em ambiente 2D e/ou 3D através de linhas. No conceito BIM, a porta em questão é uma entidade única que tem os seus elementos geométricos e propriedades intrínsecas definidas (Pereira, 2017).

Analisando estudos realizados com as duas plataformas, podemos observar as vantagens $\mathrm{e}$ desvantagens de cada um. Um estudo realizado por Campos Neto, Taveira e Moreau (2017) teve o objetivo de avaliar o diferencial ao se projetar nestas plataformas, visto que o conceito BIM. Para isso, um mesmo projeto foi desenvolvido em ambas as plataformas, a fim de demonstrar qual possui as melhores características no desenvolvimento e resultado final, apontando as dificuldades, facilidades, estética, leitura, manuseio, interface e ferramentas. Os autores concluíram ambos métodos apresentam vantagens e desvantagens, de forma que cada profissional precisa avaliar a situação, em qual plataforma vai se familiarizar melhor e se adaptar, o potencial de investimento da empresa e de todos os envolvidos na área de projeto para a escolha do software que melhor irá atender suas necessidades.

Ainda segundo Campos Neto, Taveira e Moreau (2017), a plataforma BIM apresenta funções e características de alto nível, mas necessita de um grande investimentoem equipamentos esoftwares, treinamentos dos profissionais e uma interligação com todas as outras partes envolvidas em um projeto, de modo a aproveitar todo o seu potencial. O software CAD apresenta maior confiança entre os profissionais por estar a muitos anos no mercado, a questão financeira para implantação do BIM ou ainda, de acomodação. Embora seja necessário avaliar cada situação com base em custos, treinamentos e tempo necessário para desenvolvimento dos projetos, o BIM apresenta contribuições com as quais as empresas só tendem a ganhar, um diferencial no mercado de engenharia civil.

Já o estudo realizado por Marinho (2017), realizou uma análise comparativa entre os dois métodos, tecnologia $\mathrm{CAD}$ e tecnologia BIM, no levantamento de quantitativos para elaboração de orçamento de uma residência unifamiliar e um prédio residencial, para as etapas de fundação, estrutura, alvenaria e acabamentos em geral. Foi observado que o software BIM utilizado, o Revit, levantou quantitativos com porcentagens menores que o método manual com o auxílio da ferramenta $\mathrm{CAD}$, representando diferenças orçamentárias de 3 mil reais na casa e de 300 mil reais no prédio. Dessa forma, conclui-se que mesmo em uma simples casa, o impacto financeiro é significativo.

Ainda se observou que o BIM apresentou maior rapidez no levantamento, devido ao seu processo ser praticamente automático, além da sua facilidade de trabalho e sua precisão de resultados gerados na modelagem. Também possui a vantagem de identificar 
automaticamente a ocorrência de incoerências ou falhas. A desvantagem observada foi a grande necessidade de conhecimento e familiaridade com o software e com o processo construtivo para a correta modelagem (Marinho, 2017).

Assim, em seu estudo, Marinho (2017) conclui que apesar dos investimentos iniciais mais altos e da necessidade de um maior nível de conhecimento, a utilização da tecnologia BIM pode ser mais vantajosa ao diminuir a ocorrência de erros e, consequentemente, de desperdícios de materiais, propiciar orçamentos mais enxutos e adequados, e diminuir o tempo de planejamento e prazo da obra.

A fim de demonstrar as facilidades das ferramentas BIM, Pereira (2017) desenvolveu um projeto arquitetônico de uma residência unifamiliar fictícia utilizando o Autodesk Revit Architecture 2017 versão educacional. O autor conclui que o software apresenta inúmeras vantagens, como interface gráfica intuitiva, facilidade na geração de desenhos e extração de quantitativos e criação de vistas 3D automáticas. Para utilizá-lo, o usuário precisa ter conhecimento do processo construtivo, porém na precisa ser um expert em Revit para poder manuseá-lo. O autor ainda aponta que a plataforma BIM, por ser colaborativa, aproxima todas as equipes envolvidas direta ou indiretamente no projeto, proporcionando a integração de todas as disciplinas e facilitando a visualização das inconsistências.

Em um estudo de caso, Perini (2017) realizou o acompanhamento do projeto de uma residência unifamiliar de alto padrão e de grande porte, de mais de $400 \mathrm{~m}^{2}$, com a utilização do Revit. A autora observou a ferramenta BIM foi de extrema importância para o planejamento da estrutura, uma vez que devido ao seu porte, quanto mais detalhes puderem ser extraídos dos projetos, melhor será o gerenciamento e a execução do mesmo, podendo diminuir custos com retrabalhos. $\mathrm{O}$ software atendeu de maneira eficaz a compatibilização do projeto e facilitou a execução dessa etapa.

Quanto a comparação com o tradicional AutoCAD, a autora aponta que o Revit apresenta uma melhor visualização do projeto, possibilitando uma visão em 3D de cada item necessário, além de tornar possível a remodelagem e resolução de qualquer detalhe ou conflito no mesmo instante (Perini, 2017).

Em um estudo semelhante, Silva e Comparim (2016) realizaram uma comparação entre a metodologia CAD e a metodologia BIM no desenvolvimento de um projeto de uma residência unifamiliar com a utilização dos softwares AutoCAD e Revit no âmbito do gerenciamento, planejamento e orçamento da obra. $\mathrm{Na}$ análise da compatibilização dos projetos no âmbito orçamentário, foi demonstrado através do estudo que o desenvolvimento de projetos de forma isolada através do AutoCAD e sem interação entre os projetistas, podem gerar muitos prejuízos nos empreendimentos, por mais simples e pequenos que sejam. Dessa forma, a tecnologia BIM fornece aos projetos maior precisão tanto no planejamento quanto na execução da edificação com a redução de erros e retrabalhos, minimizando os prejuízos e gerando maiores lucros para os clientes e construtores.

Em sua pesquisa, Teixeira (2018) realizou a compatibilização dos projetos estrutural arquitetônico e hidrossanitário de uma residência unifamiliar de 236,83 $\mathrm{m}^{2}$ utilizando o software Revit, da plataforma BIM, e o AutoCAD, da plataforma CAD. Foi observado que através da modelagem com o Revit identificouse incompatibilizações, gerando economia na obra e garantindo segurança estrutural, através da previsão dos furos necessários na estrutura em concreto armado ou alteração do projeto hidrossanitário. Além disso, possibilitou uma apresentação do projeto de forma mais realista para o cliente.

Observou-se ao longo de todos os estudos descritos que a tecnologia BIM apresentou inúmeras vantagens e benefícios para a realização de projetos em relação a tecnologia CAD. Ainda que o AutoCAD ainda seja o software mais utilizado para a realização dessa etapa, o Revit tem se destacado pelos benefícios que apresenta e a forma como pode diminuir custos, desperdícios, erros e falhas. A principal desvantagem encontrada está no alto investimento inicial e necessidade de conhecimentos técnicos, que podem ser minimizados ao se observar que a ferramenta oferece aumento da produtividade e redução de custos.

\section{Considerações finais}

A inserção de novas tecnologias na construção é uma ferramenta para otimizar projetos, oferecendo aos seus clientes maior segurança em seus empreendimentos. Além disso, atende a uma tendência de mercado, com consumidores cada vez mais exigente e preocupados com a diminuição de desperdícios e sustentabilidade. Assim, o desenvolvimento de tecnologias como a plataforma CAD e a plataforma BIM apresentou avanços na elaboração de projetos, cada uma no seu tempo e com suas vantagens e desvantagens.

O presente estudo buscou realizar uma análise comparativa entre os dois métodos para o projeto de residências unifamiliares, mais destacadamente para o uso dos softwares AutoCAD e Revit, através de uma revisão bibliográfica de estudos sobre o tema. Após estudos preliminares sobre cada um dos métodos e a seleção dos estudos, foi realizada a comparação dos resultados, que foram sintetizados através desse artigo.

Dessa forma, pode-se observar que a plataforma BIM apresenta vários benefícios de aplicação, em todos os tipos de prédios. Esses benefícios são ainda mais destacados em edifícios de grande porte, possibilitando 
o detalhamento da estrutura e a compatibilização de todos os projetos, com a integração das equipes. Ainda que seja em obras menores como uma residência unifamiliar, esses benefícios podem ser sentidos, uma vez que acarreta em expressiva redução de desperdícios, um projeto de maior qualidade e redução de custos, pela redução de erros e fornecimento de um projeto integrado.

O software Revit é o mais utilizado, possuindo uma interface mais clara e de uso mais simplificado. Além disso, atende às necessidades para a compatibilização de projetos e abre portas para a utilização de outras ferramentas da plataforma BIM. Seu uso ainda é pouco disseminado no Brasil, devido ao seu maior custo e maior necessidade de qualificação do profissional. Essa se apresenta como a maior desvantagem do sistema que pode ser vencida pela capacitação e atualização dos profissionais nas tecnologias disponíveis no mercado, para atender a um mercado consumidor cada vez mais exigente. Assim, essas ferramentas podem oferecer maior qualidade em seus projetos, maior produtividade e redução de custos, possivelmente maiores do que os investimentos iniciais a longo prazo.

\section{Referências}

Aparecida, G. S. (2018). Avaliação do uso da tecnologia BIM como ferramenta de fiscalização de obras públicas na etapa de concepção e elaboração de projetos (Graduação). Universidade Federal de Uberlândia.

Brito, A. N. (2017). Avaliação da modelagem BIM 5D no orçamento de obras públicas (Graduação). Universidade de Brasília.

Campos Neto, L., Taveira, A., \&Moreau, N. (2017). Desenvolvimento de projeto de edificação: um estudo comparativo nas plataformas CAD e BIM. Iberoamerican JournalOf Project Management, 8(2), 31-48. Disponível em http://www.ijopm.org/index.php/IJOPM/article/view/317

Marinho, R. (2017). Análise comparativa do levantamento de quantitativos entre o método manual e a plataforma BIM (Graduação). Universidade Federal do Ceará

Martinez, L., \& Amorim, S. (2010). Inserção de aspectos sustentáveis no projeto de arquitetura unifamiliar e capacitação de profissionais de arquitetura em Niterói. In Congresso Nacional de Excelência em Gestão (pp. 1-23). Niterói: Inovarse. Disponível em http://www.inovarse.org/node/1492

Mobus Construção. Tecnologia BIM: o que é e como aplicar em projetos de construção. (2019). Acesso em 6 de outubro 2019, disponível em https://www. mobussconstrucao.com.br/blog/tecnologia-bim-projetos-de-construcao/

Pereira, N. J. (2017) Utilização da tecnologia BIM no desenho arquitetônico: um estudo de caso (Graduado). Universidade Federal do Recôncavo da Bahia.

Perini, V. (2017) Estudo de caso: compatibilização de edificação unifamiliar de dois pavimentos de alto padrão utilizando a tecnologia BIM (Graduado). Universidade do Planalto Catarinense.

Pires, L. S. R. (2018) Extração de quantitativos com uso de BIM: estudo de caso em edificação unifamiliar (Doutor). Universidade Federal de Santa Catarina.

Silva, F. A. M. (2018) Compatibilização de projetos com uso da tecnologia $B I M$ (Graduado). Centro Universitário de Formiga.

Silva, J. L., \&Comparim, L. L. (2016) Estudo de caso: análise comparativa do orçamento e planejamento de uma residência unifamiliar utilizando as ferramentas AutoCAD e Revit (Graduado). Universidade Tecnológica Federal do Paraná.
Soliman Junior, J. (2015) Projeto de edificações com uso de BIM: potencialidade e diretrizes básicas de modelagem visando ao atendimento às especificações de desempenho (Graduado). Universidade Federal do Rio Grande do Sul.

Teixeira J. D. (2016) Compatibilização de projetos através da modelagem $3 D$ com uso de software em plataforma BIM (Graduado). Universidade Federal de Santa Catarina. 